\title{
Stimulation sweet spot in subthalamic deep brain stimulation-myth or reality? A critical review of literature
}

Alexis de Roquemaurel ${ }^{1}$, MD; Thomas Wirth ${ }^{1,2,3}$, MD; Nirosen Vijiaratnam ${ }^{1}$, MD; Francisca Ferreira ${ }^{1}$ MD; Ludvic Zrinzo ${ }^{1}$, MD, PhD, Harith Akram ${ }^{1}$ MD, PhD; Thomas Foltynie ${ }^{1}$ MD, PhD; Patricia Limousin ${ }^{1}, \mathrm{MD}, \mathrm{PhD}$

1. Unit of Functional Neurosurgery, Department of Clinical and Movement Neurosciences, UCL Queen Square Institute of Neurology and the National Hospital for Neurology and Neurosurgery

2. Neurology department, Strasbourg University Hospital, Strasbourg, France;

3. INSERM-U964/CNRS-UMR7104/University of Strasbourg, Illkirch-Graffenstaden, France

\section{Corresponding author information:}

Dr Alexis de Roquemaurel

Department of Clinical and Movement Neurosciences, UCL Queen Square Institute of Neurology and the National Hospital for Neurology and Neurosurgery

33, Queen square, London, United-Kingdom

Tel: +44 2034488741

Fax: +44203448 8642

Email: alexis.deroquemaurel@,nhs.net

Short title : Stimulation sweet spot in subthalamic deep brain stimulation-myth or reality?

Number of Tables: 5

Number of figures: 3

Manuscript words count: 4140

Abstract word count: 236

Keywords:

Parkinson's disease

Deep Brain Stimulation

Subthalamic Nucleus

Volume of Tissue Activated

Sweet spot

Conflict of interest: none

Funding source: none 


\begin{abstract}
:
Introduction: While deep brain stimulation (DBS) of the subthalamic nucleus (STN) has been extensively used for more than 20 years in Parkinson's disease (PD), the optimal area of stimulation to relieve motor symptoms remains elusive.

Objective: We aimed at localizing the sweet spot within the subthalamic region by performing a systematic review of the literature.

Method: PubMed database was searched for published studies exploring optimal stimulation location for STN DBS in PD, published between 2000 and 2019. A standardised assessment procedure based on methodological features was applied to select high quality publications. Studies conducted more than 3 months after the DBS procedure, employing lateralised scores and/or stimulation condition, and reporting the volume of tissue activated or the position of the stimulating contact within the subthalamic region were considered in the final analysis.

Results: Out of 439 references, 24 were finally retained, including 21 studies based on contact location and three studies based on VTA. Most studies (all VTA-based studies and 13 of the 21 contact-based studies) suggest the superior-lateral STN and the adjacent white matter as the optimal site for stimulation. Remaining contact-based studies were either inconclusive (5/21), favoured the caudal zona incerta $(1 / 21)$ or suggested a better outcome of STN stimulation compared to adjacent white matter stimulation $(2 / 21)$.

Conclusion: Using a standardised methodological approach, our review supports the presence of a sweet spot located within the supero-lateral STN and extending to the adjacent white matter.
\end{abstract}

\title{
Highlights:
}

The optimal area of stimulation to improve motor symptoms with STN-DBS in Parkinson's disease remains a matter of debate.

Most studies selected in our work suggest an optimal stimulation area within the supero-lateral STN subthalamic nucleus and the adjacent white matter.

Averaged coordinates of the reported active contacts corresponded to the supero-lateral and posterior subthalamic nucleus.

Future improvements in VTA prediction models, as well as better consideration for non-anatomical factors influencing the DBS outcome might further improve the delineation of this sweet spot. 


\section{Introduction}

Deep Brain Stimulation (DBS) of the Subthalamic nucleus (STN) is a well-established approach for the management of motor fluctuations in Parkinson's disease (PD) (1-6). Whilst this treatment benefits most patients, divergences in outcomes exist (7). This has brought attention to factors that might potentially explain the divergence $(7,8)$, such as electrode placement and the volume of tissue activated (VTA) by stimulation $(7,9,10)$.

The identification of an optimal stimulation area or 'sweet spot' within the subthalamic region is crucial in surgical planning. This, combined with the emergence of new programming strategies based on VTA prediction, might help reducing programming time $(11,12)$, as well as avoiding subtle or delayed onset side effects (13). Nevertheless, despite the extensive use of this concept in the literature for over twenty years, its exact location remains elusive. A systematic review aiming to address this issue (10) found large discrepancies between studies, leaving the question unanswered. Potential reasons underlying this heterogeneity include the variability in the strategies used to localise the stimulation site, the active contact positions or the location and boundaries of the STN (10). A recent survey of movement disorders specialists aiming at identifying the theoretical best contact location on atlas slices also demonstrated disagreement between experts (9).

While contact-based studies have been the mainstay of this exploration, recent advances in VTA modelling has brought novel insights into this field $(14,15)$, that can be exploited to explore the effect of different stimulation settings on the stimulation volume. A growing number of studies using this newly available strategy as a way to identify the best location of stimulation for motor outcome have been released over the last few years, prompting an update of the available evidence. Furthermore, ever since the increased reliance on visualization platforms as a tool to assist with DBS programming, the identification of the stimulation 'sweet spot' as well as 'sour spots' (for side effects) has become of great relevance to the programming clinician and not just the operating neurosurgeon. In this work, we aim to review the existing literature and apply strict methodological criteria to identify the ideal stimulation location in the STN for the treatment of PD motor symptoms. Given the increasing use of imaging software as a promising tool to assist with DBS programming, this work was a primarily focused on studies involving imaging-defined STN. 


\section{Material and methods}

A comprehensive review of the literature was conducted over the period of August to December 2019 using Medline (Pubmed) and the Cochrane Library. The search strategy included the terms (active contact) or (most effective contacts) or (sweet spots) or (localization) or (location) or volume of tissue activated) and (subthalamic nucleus) and covered the period between 01/2000 and 12/2019. No language restrictions were applied. Papers were excluded if they were not relevant to the search topic. Of the subsequent included papers, a manual search of bibliographies was conducted to identify additional articles that may have been missed on the initial search. Data regarding connectivity pattern associated with the optimal stimulation area $(7,16)$, or with the beta oscillation source $(7)$, were considered as beyond the scope of this review and therefore excluded.

Articles included were then categorised according to the method used to describe the best stimulation spot (contact location or volume of stimulation).

For studies based on contact position, the time interval between stimulation setting and clinical assessment served to differentiate the ones assessing the outcome in the short-term (contact testing and clinical assessment performed the same day) from long-term studies. Long-term studies based on contact location were further categorised between those simply reporting the position of the optimal contact as defined through clinical care and the ones further exploring the relationship between contact position and motor outcome (either through group comparison or by mapping the clinical effect of stimulation within the STN). In the text, studies falling into the first category will be mentioned as "active contact localisation studies" while the ones relying on motor outcome to statistically determine the best stimulation area will be mentioned as "motor outcome/contact location association studies".

Within each category, articles were individually reviewed using methodological criteria selected from a consensus between authors based on their deemed impact on study outcome. These included: number of patients, timing between DBS implantation and assessment, STN segmentation (imaging, atlas, MER) and lead visualisation methods (planar X rays, fluoroscopy, CT scan, MRI scan). Specific attention to these elements was emphasised in view of previous studies suggesting that imaging modality for lead detection, as well as the method to define individuals STN boundaries might influence the main findings (17-19). When atlas-based segmentation was used, the method employed (type of co-registration or transposition strategy) was noted. Similarly, when group-based analysis was performed from individual coordinates or scans, the normalisation method (transposition onto a common space) was examined. For studies assessing the motor outcome in relation to stimulation location, the method of evaluation, including the type of motor assessment, medication status during motor assessment (ON, OFF or not described), and lateralisation (total or lateralised score, under unilateral or bilateral stimulation) were recorded. For VTA based studies we also reviewed VTA 
models (FEM-based or heuristic, adapted to individual electrodes' impedances or not). Main results concerning optimal stimulation sites were noted, as well as contact or stimulation coordinates, and information regarding active contact distribution within the STN area, when available.

In order to warrant comparability between studies, we defined selection criteria based on the abovementioned features to focus the review analysis on high-quality studies. Studies enrolling less than 12 patients, early stimulation settings assessment ( $<3$ months from surgery), without STN segmentation or based on planar X rays for lead detection were consequently excluded from main results analysis. Since motor assessment relying on non-lateralized score reflects the average effect from both contacts locations on motor outcome, we purposely focused on studies using lateralized score and/or unilateral stimulation.

While this work focused on providing conclusions regarding the stimulation sweet spot on imaging, studies relying on MER-based STN segmentation and otherwise fulfilling methodological criteria were included but considered separately for further comparisons.

\section{Results}

\section{Studies}

Among the 439 articles identified, we selected 55 that fulfilled the initial selection criteria (figure 1). One article could not be accessed online (20) and another four, based on connectivity profile $(7,16)$, beta oscillation signal $(7,21)$, or exclusively studying freezing of gait and falls (22), were considered as beyond the scope of this review, and excluded. Following a review of the remaining 50 articles, 11 additional studies were identified and included in the present work and 61 (10 VTA based and 51 contact based) studies were finally used in this review.

\section{DBS contact location}

Nineteen studies reported distribution of contacts 'location (two short-term and 17 long-term, see Figure 1). The proportion of contacts in various subthalamic areas is presented in (Table 1). Six studies $(31.5 \%)$ had most contacts within the STN, four (21\%) a majority at the superior border, while $6(31.5 \%)$ had a roughly equal distribution between areas (inside/outside STN or inside/superior border/above the STN, see figure 2). Three studies (15.8\%) had most contacts above the STN (table $1)$.

\section{Excluded studies after methodological assessment}

Six out of 10 VTA based studies were excluded with the following reasons: insufficient number of participants included $(14,23,24)$, interval of time between surgery and stimulation assessment not 
reported (24), non-lateralised motor assessment with bilateral stimulation (25-28) motor assessment reported ON medication $(27,28)$ and the absence of true VTA modelling with the use a common predefined VTA size, regardless of individual stimulation settings (therefore not considering the effect of stimulation parameters in the resulting volume of stimulation) (26).

Thirty-two/51 studies were excluded. Among those, eight short-term studies were excluded due to: insufficient number of participants included (29-32) assessment of bilateral stimulation with total UPDRS-III (32-34), stimulation procedure being performed ON medication (31,34), variable/insufficient time from DBS implantation $(29,32,32)$ or time from surgery not being mentioned (31). Additionally, lead detection was based on planar X-rays in two studies $(32,32)$ and not clearly described in one (35); and 24 long-term studies were excluded due to: insufficient number of patients included (36-39), absence of STN segmentation (39-44), and use of planar X-rays for contact location $(9,37,42,45-47)$, lead localisation method not described and absence of contact location (20), motor assessment performed ON medication (48-50), medication status not specified (51) and use of bilateral motor scores to analyse bilateral stimulation $(25,39,52-56)$ (supplementary Table 1-4).

\section{VTA}

Out of ten VTA based studies reviewed, three fulfilled the methodological criteria $(15,57,58)$ (Table 2). All three studies utilized voxel analysis to identify the optimal stimulation area to the superolateral STN $(15,57,58)$ and adjacent ZI/H2 white matter, beyond the superior limit of the STN $(15,57)$.

\section{Contact based study using short term assessment}

Eleven/51 contact-based studies included short-term assessment of deep brain stimulation (Table 3). Following methodological evaluation, three/11 studies using MRI location of the STN were retained (59-61). Among the three selected studies, STN segmentation was based on an atlas, with the Mai atlas being registered linearly to patients scan using landmarks as described and validated using the centre of the red nucleus as determined by experts in Videen et al. (19).

McNeely et al. and Gourisankar et al. both compared the effect of ventral (inferior) and dorsal (superior) stimulation on motor outcomes and did not observe a significant difference apart from rigidity being improved by ventral stimulation in Gourisankar's work $(59,61)$. Eisenstein et al. and Gourisankar et al. also used a voxel-based analysis, weighting voxels according to the distance from the stimulating contact. The former did not identify a specific area associated with global motor improvement, although some lateralised symptoms were associated with specific stimulation spots (right hand bradykinesia with stimulation of the STN/ZI interface i.e superior border, and left hand tremor was associated with the STN/SN interface) (60). Gourisankar et al. further reported 
improvement associated with the stimulation of the STN (rigidity), the superior STN (bradykinesia, tremor) and the adjacent ZI (total UPDRS-III) (61).

\section{Contact-based studies using long term assessment}

Twenty-six/40 studies fell into the motor outcome/contact location association studies category while the remaining 14 were active contact localisation studies (table 4).

Of 14 active contact localisation studies, two using MRI location of the STN fulfilled the methodological criteria (62,63), while six additional MER-based studies were selected (64-69) (Table 4). Regarding the studies based on imaging-defined delineation of the STN, Vergani et al. reported a balanced contact distribution between the ZI/Forel's field H2 and the STN (62), while Connolly et al. reported that most optimal stimulation contacts were localised within the supero-lateral STN (63). Using MER-defined STN, Saint Cyr et al. described the optimal stimulation area as being in an area extending from the antero-superior STN to the adjacent white matter (ZI/ Forel's field H2) (64). Zheng et al. reported the best contact location as anteriorly and superiorly to the superior STN boundary (68), while the remaining four MER-based studies described the superior limit of the STN as being the optimal stimulation location $(65-67,69)$.

Eight motor outcome/contact location association studies using imaging-based location of the STN $(17,70-76)$ (Table 5) as well as two additional comparative MER-based studies $(8,41)$ met the methodological criteria. While Welter et al; (73) and Chazeron et al; (74) also compared total UPDRSIII with bilateral contact location, they only considered pairs of contact with similar locations, limiting the confusion of incongruent contact locations when interpreting the motor outcome, therefore these two studies were included. Most of the eight studies tended to advantage the STN $(73,74)$, and more precisely, the antero-superior STN (antero-superior STN (71), anterior-supero-lateral STN (75) and antero-lateral STN (72). Two studies did not observe any effect associated with stimulation placement $(17,76)$, although Weise et al. (17) concluded that most contacts were located within the superior half of the STN which also represented half of all contacts location in the study from Mostofi. et al. (Table 5). One study supported caudal ZI over STN stimulation (70). When considering MER-based studies, Verhagen et al. reported the postero-lateral STN as an optimal stimulation location (8). Conversely, Bot et al., by comparing the position of stimulation contacts associated with poor, satisfying and optimal responses to an electrophysiology-defined superior border of the STN did not find statistically significant differences (41).

\section{Coordinates}

Stimulation contact coordinates in relation to the AC-PC line were reported in 16 (14 contact-based studies and two VTA-based studies) of the $24(21+3)$ studies which satisfied the methodological 
review. A total of nine/16 studies, which did not indicate any normalisation of AC-PC based coordinates, were excluded $(17,41,66-71,76)$. Of note, in one of those studies the caudal ZI was selectively targeted (70).

Among the seven/16 remaining studies, the coordinate correction system (based on template dimension) varied significantly. Methods included AC-PC based correction (Saint Cyr et al. correction with AC-PC length of $23 \mathrm{~mm}$ (64)) and co-registration to common spaces, namely MNI space in Dembek et al. and Akram et al. (15,57), Mai atlas in Gourisankar et al. and Eisenstein et al. (61), Schaltenbrand and Wahren atlas in Vergani et al. (62), and a digitised version of the Morel Atlas using averaged dimension of all patients in Garcia-Garcia et al. (75). AC-PC lengths could be obtained either from the article directly or from available characteristics of common spaces for the seven studies and was used to normalise $\mathrm{x}, \mathrm{y}$ and $\mathrm{z}$ coordinates to a common AC-PC length of $23.29 \mathrm{~mm}$ (supplementary table 5). For three studies $(15,60,61)$ which expressed the antero-posterior (y) coordinate from another origin than the mid-commissural point, medio-lateral (x) and supero-inferior (dorso-ventral, z) coordinates were kept unchanged and antero-posterior coordinates were converted to MCP based coordinates from the AC-PC length for comparability (supplementary table 5). Corrected average MCP based coordinates ranged from $9 \mathrm{~mm}$ (57) to $11.9 \mathrm{~mm}$ (64) laterally, $-0.8 \mathrm{~mm}$ (62) to $-5.1 \mathrm{~mm}(61)$ posteriorly and from $0 \mathrm{~mm}(15)$ to $-2.9 \mathrm{~mm}$ inferiorly to MCP (61). Averaged values were $\mathrm{x}: 10.5 \mathrm{~mm}( \pm 1)$, y: $-2.5 \mathrm{~mm}( \pm 1.5)$, z: $-1.7 \mathrm{~mm}( \pm 0.9)$ (based on 448 contacts). Those averaged coordinates correspond to the supero-lateral and posterior STN on the MNI template (MNI coordinates: $\mathrm{x}: 11.6 \mathrm{~mm} \pm 1 \mathrm{~mm}, \mathrm{y}:-13.1 \mathrm{~mm} \pm 1.6 \mathrm{~mm}, \mathrm{z}:-6.7 \mathrm{~mm} \pm 1 \mathrm{~mm}$, Figure 3, supplementary table $5)$. 


\section{Discussion}

In this review, we extensively screened the literature contributing to the knowledge of the 'best' stimulation area for STN-DBS in PD, selecting only high-quality studies through a predefined set of standardized criteria. Our findings suggest the supero-lateral STN and its adjacent superior white matter as the most relevant candidate areas to achieve optimal motor outcome. This is supported by three rigorous VTA based studies as well as 14 contact-based studies. These results are in accordance with a previous review on best stimulation placement (10). The important role of the supero-lateral STN and the adjacent white matter tracts is further supported by the predominance of active contacts located within these regions $(17,76)$ (Table with distribution of contacts). Our findings are also in agreement with previous histological (77) and physiological studies (78) conducted on primates as well as imaging studies (79) in humans (80). Local field potential recording studies measuring beta oscillatory activity, a marker of the best stimulation area (21) also concurs with this.

Differentiating between the superior STN and the adjacent white matter when attempting to define the optimal stimulation area, has been the focus of major attention in the recent literature $(14,24,73,74)$. In our review, two of the three VTA based studies $(15,57)$ and seven $(62,64-69)$ contact-based studies suggested a positive effect of stimulation located in the ZI/H2 region including some reporting minimal or no overlap between the optimal stimulation area and the supero-lateral STN $(15,68)$. The overall variability when opposing these two areas, might be explained by methodological differences including the use of MER to define STN boundaries (24). Indeed, Weise et al. reported a mean difference of $2.8 \mathrm{~mm}$ in the length of the electrodes placed within the STN, when comparing MRI based and MER based STN delimitation. This resulted in conflicting findings between MRI and MER based segmentation, with 14\% and 50\% of active contacts being located beyond the superior border of the STN respectively (17). Further supporting this view, most reviewed studies describing a majority of contact within the STN used a MRI-based definition of the STN, while in two out of three studies describing a majority of contact above the superior limit of the STN, the superior boundary was defined by MER (Table 1).

Among other factors, overestimation of the volume of the STN on MRI (especially when using susceptibility imaging), MR image distortion in absence of adequate correction, but also error in the location of the superior border of the STN with MER resulting from brain-shift, or due to suboptimal trajectory angle missing the most superior part of the STN, might contribute to the observed discrepancy between MRI and MER.

Noteworthy, the variety of motor symptoms associated with PD, could further explain the extension of the optimal stimulation area from the superior-lateral STN to the adjacent ZI/H2 area. Indeed, several authors have reported that the different motor features of PD might respond optimally to 
different stimulation area, albeit with conflicting results. According to Cintas et al., the best contact was common for both akinesia and rigidity in only $41 \%$ of cases (65). Moreover, Gourisankar et al. describes that tremor and bradykinesia were better improved by stimulation of the superior STN, while rigidity responded to the stimulation of the whole STN, and possibly more within its inferior part (61). Furthermore, some authors have also suggested different mechanisms underlying tremor control between proper STN stimulation and ZI stimulation (36). Other studies bring more moderate conclusions with some degree of overlap between symptom specific sweet spots. For Butson et al. (14), bradykinesia and rigidity both responded to the stimulation in a shared area (white matter superior to STN, but with the area associated with improvement in bradykinesia more restricted than for rigidity), while Akram et al. (57) found a common area for tremor, rigidity and bradykinesia within the supero-lateral STN with some further extension medially and posteriorly for rigidity and bradykinesia. This is reinforced by Dembek et al suggesting a significant overlap in the sweet spot for rigidity and akinesia (15).

Assessment timing might also influence the resulting optimal stimulation area, as illustrated in the study from Dembek et al (15). Based on short-term motor assessment the authors described the sweet spot as mostly overlapping within the ZI/H2 area, while the long-term benefits of DBS were predicted by the degree of overlap between the VTA and the whole STN.

In view of the above observations, we believe the current literature cannot discriminate between the supero-lateral STN and its adjacent white matter tracts, which might be considered as a unit rather than two distinct targets. This hypothesis is further supported by the presence of relevant fibres tracts within the ZI/Forel's field H2 region, including afferent fibres from the motor cortex (hyperdirect pathway), and the lenticular fasciculus afferent from the globus pallidus, which have been proposed as playing a critical role in the symptoms of PD $(14,15,38,57,70,81)$.

When considering the extensive literature, inter-individual variability of motor outcomes was suggested by several studies. Indeed, this was directly described by Maks et al (24), who observed different outcomes associated with the same area of stimulation and by Schlaier et al. (50), who noted that, despite an overall better outcome associated with the stimulation of the supero-lateral STN, some patients performed better with the stimulation located in other parts of the STN. Additionally, an important dispersion was observed for contact coordinates within studies, even when considering those which employed normalisation methods: Saint Cyr et al. SD z axis 1,76mm, SD y axis 1,98mm (56 contacts) (64), Garcia-Garcia et al. SD y axis 1.93 (61 contacts) (75). This inter-individual variability might explain the limited results of purely model based programming approach $(11,13)$, and while the precise reason for this heterogeneity remains to be explored, this might challenge the concept of a uniform sweet post applicable to all PD patients, regardless of clinical, imaging or genetic 
characteristics. Inter-individual differences in STN size, shape, functional organisation and connectivity profile will surely need further consideration and dedicated investigations.

To our knowledge, our study is also the first critical review of recent VTA-based studies, which potentially provide a superior understanding of the anatomical-stimulation correlation of outcomes. Indeed, contact-based strategies only bring an indirect estimate of the most suitable stimulation spot for STN DBS, as the location of the active contact does not comprehensively explore the entire subthalamic area volume exposed to stimulation, but only the electrode trajectory. Hence, the position of the optimal contact is universally associated to the lead trajectory, thus binding the position of the stimulation along the antero-posterior, medio-lateral and supero-inferior axis. Therefore, one cannot rule out that an apparent better outcome with superior stimulation is not actually driven by an associated lateral or anterior position along the lead trajectory. Conversely, VTA models, when combined with voxel-based analysis, allow the exploration of smaller or larger volumes. This, along with the possibility to predict the spread of stimulation resulting from directional contacts will undoubtedly improve the definition of the optimal stimulation area $(15,58)$. These two factors might explain why all selected studies based on VTA prediction reached similar conclusions as opposed to more discrepant findings from contact-based studies. However, VTA approaches also carry important limitations. Indeed, most models are based on predicting the depolarisation of a myelinated axon of a predefined diameter and electric properties and oriented perpendicular to the direction of the electric field vector (82). Therefore, they only provide partial information about the effect of stimulation on surrounding neural elements. While some models have been validated against EMG recording for the prediction of the stimulation of the pyramidal tracts coursing within the internal capsule, the ability of such models to predict the effect of the stimulation on other tracts such as the hyper direct pathway or the neuronal bodies within the STN remains to be demonstrated $(23,82)$. Furthermore, such methods provide a binary representation where every voxels located within the VTA are considered as being equally affected by the stimulation, regardless of the position of this voxel within this volume $(15,57,58)$. This could potentially limit the accuracy of voxel-based analysis. Despite those inherent limitations, constant progress such as the inclusion of tissue anisotropy $(14,23,24)$, tissue inhomogeneity $(15,58)$, axon characteristics (83) as well as stimulation conditions beyond simply stimulation amplitude (84) will allow further improvements in the reliability of this approach.

Although our approach accounts for the differences of mainstream methodological contributions to localizing stimulation within the STN area, other aspects of the process, such as co-registration accuracy, the use of templates and landmarks, differences in rater segmentation expertise, imaging quality, and VTA modelling differences are important in the interpretation of the results. While most studies involved a limited number of participants (median 25 patients), which restricts the interpretation of the reported findings, very small sample-based studies were excluded (less than 12 
patients, corresponding to less than $20 \%$ of all studies) in order to limit this potential bias. A further confounding point is the inclusion of studies with an array of evaluation timing. Although the value of the short effect in predicting long-term outcomes remains unclear, we decided to keep studies that tested the short-term effect of stimulation for a number of reasons. Firstly, it is worth mentioning that the magnitude of the benefit from DBS might vary between patients due to factors independent of the stimulation area (7). Therefore, a given outcome can represent, depending on the patient, either the optimal effect (best possible effect), or to the contrary a suboptimal effect, which could be improved by moving the stimulation towards another area. In this respect, while the majority of studies based on long-term outcomes compared groups of patients, short-term studies provide direct comparison of different contact positions/VTAs within the same patient, thus accounting for inter-individual variability. Secondly, two studies that reassessed the effect of each contact during follow-up, reported an overall agreement between the best acute stimulation contact and the contact used for chronic stimulation $(47,65)$.

While we have explored stimulation positions in detail here, previous studies assessing the correlation between adequate stimulation placement and motor outcomes only suggested moderate associations $(8,15,72,75)$. These values were comparable to the ones reported by Horn et al. (7), who, could predict $26 \%$ of the variance in the observed motor improvement by combining connectivity data and VTA modelling. This, in association with the finding of an improvement in model predictability of outcomes when clinical factors were combined to connectivity-based model, highlights the necessity for adding relevant clinical and biological/genetic data to current imaging aspects for future prediction models.

To conclude, this critical review of literature incorporates the growing use of VTA-based approaches and introduces a standardised methodological method to approach the extensive and highly heterogeneous literature about the optimal stimulation area within the STN region. Our finding supports the presence of a sweet spot located within the supero-lateral STN and extending to the adjacent ZI/Forel's field H2. These findings could serve as a basis for a necessarily more tailored approach to reach the best possible outcome from STN DBS in PD patients. The contribution of individual factors, such as disease phenotype and the functional organisation of the STN, will warrant more attention in future work.

\section{$\underline{\text { Authors' Role: }}$}

AdR: organisation and execution of the review, data collection and writing; TW: organisation, review and critique; NV: organisation, review and critique; FF: execution of Figure 3; HA: review and 
critique; TF: review and critique; LZ: review and critique; PL: conception of the research project, review and critique

\section{Financial Disclosure:}

AdR's fellowship position has been financially supported by a grant from Boston Scientific. TW's fellowship was funded by two grants from the "APTES" and the "Fondation Planiol" organizations and received research grants from the Revue Neurologique and travel funding from LVL Medical. 


\section{REFERENCES}

1. Limousin P, Pollak P, Benazzouz A, Hoffmann D, Le Bas JF, Broussolle E, et al. Effect of parkinsonian signs and symptoms of bilateral subthalamic nucleus stimulation. Lancet. 1995 Jan 14;345(8942):91-5.

2. Krack P, Batir A, Van Blercom N, Chabardes S, Fraix V, Ardouin C, et al. Five-Year Follow-up of Bilateral Stimulation of the Subthalamic Nucleus in Advanced Parkinson's Disease. New England Journal of Medicine. 2003 Nov 13;349(20):1925-34.

3. Deuschl G, Schade-Brittinger C, Krack P, Volkmann J, Schäfer H, Bötzel K, et al. A Randomized Trial of Deep-Brain Stimulation for Parkinson's Disease. New England Journal of Medicine. 2006 Aug 31;355(9):896-908.

4. Weaver FM, Follett K, Stern M, Hur K, Harris C, Marks WJ, et al. Bilateral deep brain stimulation vs best medical therapy for patients with advanced Parkinson disease: a randomized controlled trial. JAMA. 2009 Jan 7;301(1):63-73.

5. Williams A, Gill S, Varma T, Jenkinson C, Quinn N, Mitchell R, et al. Deep brain stimulation plus best medical therapy versus best medical therapy alone for advanced Parkinson's disease (PD SURG trial): a randomised, open-label trial. Lancet Neurol. 2010 Jun;9(6):581-91.

6. Okun MS. Deep-Brain Stimulation for Parkinson's Disease. New England Journal of Medicine. 2012 Oct 18;367(16):1529-38.

7. Horn A, Neumann W-J, Degen K, Schneider G-H, Kühn AA. Toward an electrophysiological "sweet spot" for deep brain stimulation in the subthalamic nucleus. Hum Brain Mapp. 2017 Jul;38(7):3377-90.

8. Verhagen R, Bour LJ, Odekerken VJJ, van den Munckhof P, Schuurman PR, de Bie RMA. Electrode Location in a Microelectrode Recording-Based Model of the Subthalamic Nucleus Can Predict Motor Improvement After Deep Brain Stimulation for Parkinson's Disease. Brain Sci. 2019 Mar 1;9(3).

9. Hamel W, Köppen JA, Alesch F, Antonini A, Barcia JA, Bergman H, et al. Targeting of the Subthalamic Nucleus for Deep Brain Stimulation: A Survey Among Parkinson Disease Specialists. World Neurosurg. 2017 Mar;99:41-6.

10. Caire F, Ranoux D, Guehl D, Burbaud P, Cuny E. A systematic review of studies on anatomical position of electrode contacts used for chronic subthalamic stimulation in Parkinson's disease. Acta Neurochir (Wien). 2013 Sep;155(9):1647-54; discussion 1654.

11. Pavese N, Tai YF, Yousif N, Nandi D, Bain PG. Traditional Trial and Error versus Neuroanatomic 3-Dimensional Image Software-Assisted Deep Brain Stimulation Programming in Patients with Parkinson Disease. World Neurosurg. 2020 Feb;134:e98-102.

12. Pourfar MH, Mogilner AY, Farris S, Giroux M, Gillego M, Zhao Y, et al. Model-Based Deep Brain Stimulation Programming for Parkinson's Disease: The GUIDE Pilot Study. Stereotact Funct Neurosurg. 2015;93(4):231-9.

13. Frankemolle AMM, Wu J, Noecker AM, Voelcker-Rehage C, Ho JC, Vitek JL, et al. Reversing cognitive-motor impairments in Parkinson's disease patients using a computational modelling approach to deep brain stimulation programming. Brain. 2010 Mar;133(3):746-61. 
14. Butson CR, Cooper SE, Henderson JM, Wolgamuth B, McIntyre CC. Probabilistic analysis of activation volumes generated during deep brain stimulation. Neuroimage. $2011 \mathrm{Feb}$ 1;54(3):2096-104.

15. Dembek TA, Roediger J, Horn A, Reker P, Oehrn C, Dafsari HS, et al. Probabilistic sweet spots predict motor outcome for deep brain stimulation in Parkinson disease. Ann Neurol. 2019;86(4):527-38.

16. Vanegas-Arroyave N, Lauro PM, Huang L, Hallett M, Horovitz SG, Zaghloul KA, et al. Tractography patterns of subthalamic nucleus deep brain stimulation. Brain. 2016 Apr;139(Pt 4):1200-10.

17. Weise LM, Seifried C, Eibach S, Gasser T, Roeper J, Seifert V, et al. Correlation of Active Contact Positions with the Electrophysiological and Anatomical Subdivisions of the Subthalamic Nucleus in Deep Brain Stimulation. SFN. 2013;91(5):298-305.

18. Dormont D, Seidenwurm D, Galanaud D, Cornu P, Yelnik J, Bardinet E. Neuroimaging and deep brain stimulation. AJNR Am J Neuroradiol. 2010 Jan;31(1):15-23.

19. Videen TO, Campbell MC, Tabbal SD, Karimi M, Hershey T, Perlmutter JS. Validation of a Fiducial-Based Atlas Localization Method for Deep Brain Stimulation Contacts in the Area of the Subthalamic Nucleus. J Neurosci Methods. 2008 Mar 15;168(2):275-81.

20. Seddighi AS, Seddighi A, Nikouei A. Deep brain nucleus targeting in Parkinson's disease and essential tremor by image guided surgery using neuronavigation system with tractography and volume of tissue of activated assessment. Hell J Nucl Med. 2017 Dec;20 Suppl:14-9.

21. Zaidel A, Spivak A, Grieb B, Bergman H, Israel Z. Subthalamic span of beta oscillations predicts deep brain stimulation efficacy for patients with Parkinson's disease. Brain. 2010 Jul;133(Pt 7):2007-21.

22. Karachi C, Cormier-Dequaire F, Grabli D, Lau B, Belaid H, Navarro S, et al. Clinical and anatomical predictors for freezing of gait and falls after subthalamic deep brain stimulation in Parkinson's disease patients. Parkinsonism Relat Disord. 2019;62:91-7.

23. Butson CR, Cooper SE, Henderson JM, McIntyre CC. Patient-specific analysis of the volume of tissue activated during deep brain stimulation. Neuroimage. 2007 Jan 15;34(2):661-70.

24. Maks CB, Butson CR, Walter BL, Vitek JL, McIntyre CC. Deep brain stimulation activation volumes and their association with neurophysiological mapping and therapeutic outcomes. J Neurol Neurosurg Psychiatry. 2009 Jun;80(6):659-66.

25. Conrad EC, Mossner JM, Chou KL, Patil PG. Atlas-Independent, Electrophysiological Mapping of the Optimal Locus of Subthalamic Deep Brain Stimulation for the Motor Symptoms of Parkinson Disease. Stereotact Funct Neurosurg. 2018;96(2):91-9.

26. Haegelen C, Baumgarten C, Houvenaghel J-F, Zhao Y, Péron J, Drapier S, et al. Functional atlases for analysis of motor and neuropsychological outcomes after medial globus pallidus and subthalamic stimulation. PLOS ONE. 2018 Jul 13;13(7):e0200262.

27. Mosley PE, Smith D, Coyne T, Silburn P, Breakspear M, Perry A. The site of stimulation moderates neuropsychiatric symptoms after subthalamic deep brain stimulation for Parkinson's disease. Neuroimage Clin. 2018;18:996-1006. 
28. Petry-Schmelzer JN, Krause M, Dembek TA, Horn A, Evans J, Ashkan K, et al. Non-motor outcomes depend on location of neurostimulation in Parkinson's disease. Brain. 2019 01;142(11):3592-604.

29. Yelnik J, Damier P, Demeret S, Gervais D, Bardinet E, Bejjani B-P, et al. Localization of stimulating electrodes in patients with Parkinson disease by using a three-dimensional atlasmagnetic resonance imaging coregistration method. J Neurosurg. 2003 Jul;99(1):89-99.

30. Hilliard JD, Frysinger RC, Elias WJ. Effective subthalamic nucleus deep brain stimulation sites may differ for tremor, bradykinesia and gait disturbances in Parkinson's disease. Stereotact Funct Neurosurg. 2011;89(6):357-64.

31. Burrows AM, Ravin PD, Novak P, Peters MLB, Dessureau B, Swearer J, et al. Limbic and motor function comparison of deep brain stimulation of the zona incerta and subthalamic nucleus. Neurosurgery. 2012 Mar;70(1 Suppl Operative):125-30; discussion 130-131.

32. Yokoyama T, Sugiyama K, Nishizawa S, Yokota N, Ohta S, Akamine S, et al. The Optimal Stimulation Site for Chronic Stimulation of the Subthalamic Nucleus in Parkinson's Disease. SFN. 2001;77(1-4):61-7.

33. Tripoliti E, Zrinzo L, Martinez-Torres I, Tisch S, Frost E, Borrell E, et al. Effects of contact location and voltage amplitude on speech and movement in bilateral subthalamic nucleus deep brain stimulation. Mov Disord. 2008 Dec 15;23(16):2377-83.

34. Greenhouse I, Gould S, Houser M, Hicks G, Gross J, Aron AR. Stimulation at dorsal and ventral electrode contacts targeted at the subthalamic nucleus has different effects on motor and emotion functions in Parkinson's disease. Neuropsychologia. 2011 Feb;49(3):528-34.

35. Lanotte MM, Rizzone M, Bergamasco B, Faccani G, Melcarne A, Lopiano L. Deep brain stimulation of the subthalamic nucleus: anatomical, neurophysiological, and outcome correlations with the effects of stimulation. J Neurol Neurosurg Psychiatry. 2002 Jan;72(1):538.

36. Kitagawa M, Murata J, Uesugi H, Kikuchi S, Saito H, Tashiro K, et al. Two-year follow-up of chronic stimulation of the posterior subthalamic white matter for tremor-dominant Parkinson's disease. Neurosurgery. 2005 Feb;56(2):281-9; discussion 281-289.

37. Caire F, Derost P, Coste J, Bonny J-M, Durif F, Frenoux E, et al. [Subthalamic deep brain stimulation for severe idiopathic Parkinson's disease. Location study of the effective contacts]. Neurochirurgie. 2006 Feb;52(1):15-25.

38. Vassal F, Dilly D, Boutet C, Bertholon F, Charier D, Pommier B. White matter tracts involved by deep brain stimulation of the subthalamic nucleus in Parkinson's disease: a connectivity study based on preoperative diffusion tensor imaging tractography. British Journal of Neurosurgery. 2019 Dec 13;0(0):1-9.

39. McClelland S, Ford B, Senatus PB, Winfield LM, Du YE, Pullman SL, et al. Subthalamic stimulation for Parkinson disease: determination of electrode location necessary for clinical efficacy. Neurosurg Focus. 2005 Nov 15;19(5):E12.

40. Starr PA, Christine CW, Theodosopoulos PV, Lindsey N, Byrd D, Mosley A, et al. Implantation of deep brain stimulators into the subthalamic nucleus: technical approach and magnetic resonance imaging-verified lead locations. J Neurosurg. 2002 Aug;97(2):370-87. 
41. Bot M, Schuurman PR, Odekerken VJJ, Verhagen R, Contarino FM, De Bie RMA, et al. Deep brain stimulation for Parkinson's disease: defining the optimal location within the subthalamic nucleus. J Neurol Neurosurg Psychiatry. 2018;89(5):493-8.

42. Nowinski WL, Belov D, Pollak P, Benabid A-L. Statistical analysis of 168 bilateral subthalamic nucleus implantations by means of the probabilistic functional atlas. Neurosurgery. 2005 Oct;57(4 Suppl):319-30; discussion 319-330.

43. Guehl D, Edwards R, Cuny E, Burbaud P, Rougier A, Modolo J, et al. Statistical determination of the optimal subthalamic nucleus stimulation site in patients with Parkinson disease. J Neurosurg. 2007 Jan;106(1):101-10.

44. Nestor KA, Jones JD, Butson CR, Morishita T, Jacobson CE, Peace DA, et al. Coordinatebased lead location does not predict Parkinson's disease deep brain stimulation outcome. PLoS ONE. 2014;9(4):e93524.

45. Godinho F, Thobois S, Magnin M, Guenot M, Polo G, Benatru I, et al. Subthalamic nucleus stimulation in Parkinson's disease : anatomical and electrophysiological localization of active contacts. J Neurol. 2006 Oct;253(10):1347-55.

46. Voges J, Volkmann J, Allert N, Lehrke R, Koulousakis A, Freund H-J, et al. Bilateral highfrequency stimulation in the subthalamic nucleus for the treatment of Parkinson disease: correlation of therapeutic effect with anatomical electrode position. Journal of Neurosurgery. 2002 Feb 1;96(2):269-79.

47. Herzog J, Fietzek U, Hamel W, Morsnowski A, Steigerwald F, Schrader B, et al. Most effective stimulation site in subthalamic deep brain stimulation for Parkinson's disease. Mov Disord. 2004 Sep;19(9):1050-4.

48. Nakano N, Taneda M, Watanabe A, Kato A. Computed Three-Dimensional Atlas of Subthalamic Nucleus and Its Adjacent Structures for Deep Brain Stimulation in Parkinson's Disease. ISRN Neurol [Internet]. 2012 Jan 12 [cited 2020 May 19];2012. Available from: https://www.ncbi.nlm.nih.gov/pmc/articles/PMC3265216/

49. Sarnthein J, Péus D, Baumann-Vogel H, Baumann CR, Sürücü O. Stimulation sites in the subthalamic nucleus projected onto a mean 3-D atlas of the thalamus and basal ganglia. Acta Neurochir (Wien). 2013 Sep;155(9):1655-60.

50. Schlaier JR, Hanson C, Janzen A, Fellner C, Hochreiter A, Proescholdt M, et al. Deep brain stimulation in Parkinson's disease: motor effects relative to the MRI-defined STN. Neurosurg Rev. 2014 Jul;37(3):461-70; discussion 470-471.

51. Kasasbeh A, Abulseoud OA, Matsumoto JY, Stead SM, Goerss SJ, Klassen BT, et al. Lack of differential motor outcome with subthalamic nucleus region stimulation in Parkinson's disease. Journal of Clinical Neuroscience. 2013 Nov 1;20(11):1520-6.

52. York MK, Wilde EA, Simpson R, Jankovic J. Relationship between neuropsychological outcome and DBS surgical trajectory and electrode location. Journal of the Neurological Sciences. 2009 Dec 15;287(1):159-71.

53. Lalys F, Haegelen C, Maroua M, Drapier S, Verin M, Jannin P. Anatomo-clinical atlases correlate clinical data and electrode contact coordinates: application to subthalamic deep brain stimulation. Journal of Neuroscience Methods. 2013 Jan;212(2):297-307. 
54. Koivu M, Huotarinen A, Scheperjans F, Laakso A, Kivisaari R, Pekkonen E. Motor outcome and electrode location in deep brain stimulation in Parkinson's disease. Brain Behav. 2018;8(7):e01003.

55. Dafsari HS, Petry-Schmelzer JN, Ray-Chaudhuri K, Ashkan K, Weis L, Dembek TA, et al. Non-motor outcomes of subthalamic stimulation in Parkinson's disease depend on location of active contacts. Brain Stimul. 2018 Aug;11(4):904-12.

56. Paek SH, Yun JY, Song SW, Kim IK, Hwang JH, Kim JW, et al. The clinical impact of precise electrode positioning in STN DBS on three-year outcomes. J Neurol Sci. 2013 Apr 15;327(12):25-31.

57. Akram H, Sotiropoulos SN, Jbabdi S, Georgiev D, Mahlknecht P, Hyam J, et al. Subthalamic deep brain stimulation sweet spots and hyperdirect cortical connectivity in Parkinson's disease. Neuroimage. 2017;158:332-45.

58. Nguyen TAK, Nowacki A, Debove I, Petermann K, Tinkhauser G, Wiest R, et al. Directional stimulation of subthalamic nucleus sweet spot predicts clinical efficacy: Proof of concept. Brain Stimul. 2019 Oct;12(5):1127-34.

59. McNeely ME, Hershey T, Campbell MC, Tabbal SD, Karimi M, Hartlein JM, et al. Effects of deep brain stimulation of dorsal versus ventral subthalamic nucleus regions on gait and balance in Parkinson's disease. J Neurol Neurosurg Psychiatry. 2011 Nov;82(11):1250-5.

60. Eisenstein SA, Koller JM, Black KD, Campbell MC, Lugar HM, Ushe M, et al. Functional anatomy of subthalamic nucleus stimulation in Parkinson disease. Annals of Neurology. 2014;76(2):279-95.

61. Gourisankar A., Eisenstein S. A., Trapp N. T., Koller J. M., Campbell M. C., Ushe M., et al. (2018). Mapping movement, mood, motivation and mentation in the subthalamic nucleus. $R$. Soc. Open Sci. 5:171177.

62. Vergani F, Landi A, Antonini A, Parolin M, Cilia R, Grimaldi M, et al. Anatomical identification of active contacts in subthalamic deep brain stimulation. Surg Neurol. 2007 Feb;67(2):140-6; discussion 146-147.

63. Connolly PJ, Halpern CH, Baltuch GH, Danish SF, Jaggi JL. Implications for programming strategy of the location of the active contact in subthalamic nucleus deep brain stimulation. $\mathrm{J}$ Clin Neurosci. 2012 Jul;19(7):1029-31.

64. Saint-Cyr JA, Hoque T, Pereira LCM, Dostrovsky JO, Hutchison WD, Mikulis DJ, et al. Localization of clinically effective stimulating electrodes in the human subthalamic nucleus on magnetic resonance imaging. J Neurosurg. 2002 Nov;97(5):1152-66.

65. Cintas P, Simonetta-Moreau M, Ory F, Brefel-Courbon C, Fabre N, Chaynes P, et al. Deep brain stimulation for parkinson's disease: correlation between intraoperative subthalamic nucleus neurophysiology and most effective contacts. Stereotact Funct Neurosurg. 2003;80(14):108-13.

66. Zonenshayn M, Sterio D, Kelly PJ, Rezai AR, Beric A. Location of the active contact within the subthalamic nucleus (STN) in the treatment of idiopathic Parkinson's disease. Surg Neurol. 2004 Sep;62(3):216-25; discussion 225-226.

67. Pollo C, Vingerhoets F, Pralong E, Ghika J, Maeder P, Meuli R, et al. Localization of 
electrodes in the subthalamic nucleus on magnetic resonance imaging. Journal of Neurosurgery. 2007 Jan 1;106(1):36-44.

68. Zheng Z, Zhang Y, Li J, Zhang X, Zhuang P, Li Y. Subthalamic deep brain stimulation for Parkinson's disease: correlation of active contacts and electrophysiologically mapped subthalamic nucleus. Chin Med J. 2009 Oct 20;122(20):2419-22.

69. Guo S, Zhuang P, Hallett M, Zheng Z, Zhang Y, Li J, et al. Subthalamic deep brain stimulation for Parkinson's disease: correlation between locations of oscillatory activity and optimal site of stimulation. Parkinsonism Relat Disord. 2013 Jan;19(1):109-14.

70. Plaha P, Ben-Shlomo Y, Patel NK, Gill SS. Stimulation of the caudal zona incerta is superior to stimulation of the subthalamic nucleus in improving contralateral parkinsonism. Brain. 2006 Jul;129(Pt 7):1732-47.

71. Johnsen EL, Sunde N, Mogensen PH, Ostergaard K. MRI verified STN stimulation site--gait improvement and clinical outcome. Eur J Neurol. 2010 May;17(5):746-53.

72. Wodarg F, Herzog J, Reese R, Falk D, Pinsker MO, Steigerwald F, et al. Stimulation site within the MRI-defined STN predicts postoperative motor outcome. Mov Disord. 2012 Jun;27(7):874-9.

73. Welter M-L, Schüpbach M, Czernecki V, Karachi C, Fernandez-Vidal S, Golmard J-L, et al. Optimal target localization for subthalamic stimulation in patients with Parkinson disease. Neurology. 2014 Apr 15;82(15):1352-61.

74. de Chazeron I, Pereira B, Chereau-Boudet I, Durif F, Lemaire JJ, Brousse G, et al. Impact of localisation of deep brain stimulation electrodes on motor and neurobehavioural outcomes in Parkinson's disease. J Neurol Neurosurg Psychiatry. 2016;87(7):758-66.

75. Garcia-Garcia D, Guridi J, Toledo JB, Alegre M, Obeso JA, Rodríguez-Oroz MC. Stimulation sites in the subthalamic nucleus and clinical improvement in Parkinson's disease: a new approach for active contact localization. J Neurosurg. 2016;125(5):1068-79.

76. Mostofi A, Evans JM, Partington-Smith L, Yu K, Chen C, Silverdale MA. Outcomes from deep brain stimulation targeting subthalamic nucleus and caudal zona incerta for Parkinson's disease. npj Parkinson's Disease. 2019 Aug 21;5(1):1-7.

77. Haynes WIA, Haber SN. The Organization of Prefrontal-Subthalamic Inputs in Primates Provides an Anatomical Substrate for Both Functional Specificity and Integration: Implications for Basal Ganglia Models and Deep Brain Stimulation. J Neurosci. 2013 Mar 13;33(11):4804-14.

78. Wichmann T, Bergman H, DeLong MR. The primate subthalamic nucleus. I. Functional properties in intact animals. J Neurophysiol. 1994 Aug;72(2):494-506.

79. Accolla E, Caputo E, Cogiamanian F, Tamma F, Mrakic-Sposta S, Marceglia S, et al. Gender differences in patients with Parkinson's disease treated with subthalamic deep brain stimulation. Mov Disord. 2007 Jun 15;22(8):1150-6.

80. Hamani C, Richter E, Schwalb JM, Lozano AM. Bilateral subthalamic nucleus stimulation for Parkinson's disease: a systematic review of the clinical literature. Neurosurgery. 2005 Jun;56(6):1313-21; discussion 1321-1324. 
81. Miocinovic S, Parent M, Butson CR, Hahn PJ, Russo GS, Vitek JL, et al. Computational analysis of subthalamic nucleus and lenticular fasciculus activation during therapeutic deep brain stimulation. J Neurophysiol. 2006 Sep;96(3):1569-80.

82. Gunalan K, Howell B, McIntyre CC. Quantifying axonal responses in patient-specific models of subthalamic deep brain stimulation. NeuroImage. 2018 May 15;172:263-77.

83. Chaturvedi A, Luján JL, McIntyre CC. Artificial neural network based characterization of the volume of tissue activated during deep brain stimulation. J Neural Eng. 2013 Oct;10(5):056023.

84. Mädler B, Coenen VA. Explaining clinical effects of deep brain stimulation through simplified target-specific modeling of the volume of activated tissue. AJNR Am J Neuroradiol. 2012 Jun;33(6):1072-80.

\section{Figures Legends}

Figure 1: Flow chart

Figure 2: distribution of contacts location

Figure 3: MNI space representing the average coordinates of the optimal stimulation contact / area for each of position of the seven studies reporting corrected coordinates. The supero-inferior $(\mathrm{Z})$, anterior-posterior $(\mathrm{Y})$ and medial-lateral $(\mathrm{X})$ coordinates of the slices from the MNI space are indicated. Each plot represents a study are plotted on a common MNI space (red, Gourisankar et al.; pink, Akram et al.; bright blue, Saint-Cyr et al.; dark blue, Garcia-Garcia et al; green, Eisenstein et al.; yellow, Vergani et al.; orange, Dembek et al). 